P. D. Knoester · S. V. Belitser · C. L. P. Deckers

A. Keyser - W. O. Renier - A. C. G. Egberts

Y. A. Hekster

\title{
Diffusion of the new antiepileptic drug lamotrigine in Dutch clinical practice
}

Received: 5 July 2004/ Accepted: 7 September 2004/Published online: 20 November 2004

(C) Springer-Verlag 2004

\begin{abstract}
Introduction: Lamotrigine is one of the recently introduced antiepileptic drugs (AEDs) licensed in the Netherlands in 1995. The objective of this study was to examine the diffusion of lamotrigine into clinical practice. Three different aspects of this diffusion process were examined: incidence of use, patient characteristics and changes in prescription patterns in the first 5 years following its introduction.

Methods: A retrospective follow-up study has been conducted using drug prescription data from the database of the Dutch Drug Information Project (GIP database). Patients were included who started with lamotrigine, carbamazepine, phenytoin or valproate in the period between January 1996 and December 2000 . Incidence of use was calculated for the four drugs. Multiple logistic regression analysis was used to determine differences in baseline characteristics. The Chisquare test was used to analyse changes in the usage patterns of lamotrigine.

Results: The study population consisted of a total of 29,718 patients who were prescribed carbamazepine, phenytoin, valproate or lamotrigine for the first time in the study period. Carbamazepine and valproate accounted for the majority of all new prescriptions; the
\end{abstract}

P. D. Knoester $(\bowtie) \cdot$ C. L. P. Deckers · Y. A. Hekster

Department of Clinical Pharmacy,

University Medical Centre Nijmegen, Nijmegen,

The Netherlands

E-mail: p.knoester@erasmusmc.nl

Fax: + 31-10-4366605

S. V. Belitser · A. C. G. Egberts

Department of Pharmacoepidemiology and Pharmacotherapy,

Utrecht Institute for Pharmaceutical Sciences (UIPS), Utrecht,

The Netherlands

C. L. P. Deckers

Dutch Epilepsy Clinics Foundation, Zwolle,

The Netherlands

A. Keyser · W. O. Renier

Department of Neurology,

University Medical Centre Nijmegen, Nijmegen,

The Netherlands incidence of lamotrigine use remained stable with 4.4 patients per 100,000 per year. Baseline characteristics of lamotrigine differed depending on the patient's age and gender (OR 3.7, 95\% CI 3.3-4.2; OR 1.4, 95\% CI 1.3 1.5) relative to the conventional AEDs. In a large majority of cases, lamotrigine was used as a second-line or third-line AED. Physicians prescribing lamotrigine were predominantly neurologists, in contrast to prescribers of conventional AEDs. The prevalence of psychotropic medication and migraine-abortive drugs was significantly lower in users of lamotrigine than in users of conventional AEDs. During follow-up, several significant trends were noticed in the prescribing of lamotrigine with regard to age groups, gender, antiepileptic history and off-label use.

Discussion: Lamotrigine is prescribed to a population different from that using conventional AEDs. The uptake of lamotrigine in clinical practice is slow, for reasons probably related to characteristics of the drug itself and the prescribers. During the observation period, lamotrigine diffused gradually towards more first-line use as an AED and more off-label use.

\section{Introduction}

The field of antiepileptic drug therapy is dominated by conventional drugs, such as phenytoin (PHT; introduced in 1938), carbamazepine (CBZ; 1964) and valproate (VPA; 1971) [1]. As a substantial proportion of the patients is not controlled optimally or suffers from bothersome or clinically severe side effects while using conventional AEDs [2], there remains a clear need for new drugs. Lamotrigine (LTG) is one of the new treatment options that has been introduced in the past decade. On the basis of results from placebo-controlled trials (RCTs), LTG received regulatory approval in the Netherlands for indication in 1995 as an add-on drug in patients with refractory, localisation-related epilepsy. The reimbursement of the drug was not immediately 
approved by the Dutch Health Care Insurance Board (CVZ) because of its relatively high cost compared with conventional AEDs, and also because of a lack of favourable clinical documentation (as head-to-head comparisons with other AEDs were lacking). In August 1997, CVZ decided that the reimbursement of LTG should be restricted to the initial indication, i.e. add-on therapy for patients with refractory epilepsy. A prescription guideline, the first in the Netherlands, was issued by CVZ and distributed among Dutch neurologists (but not other physicians) to ensure the restricted use of LTG. It is to be expected, however, that once physicians become familiar with the use and safety of a new AED, the drug will be prescribed for a broader range of indications, e.g. to patients with less severe epilepsy or to patients from other age groups than those included in the initial trials. Still little is known about this process of diffusion of new drugs in daily practice. Most available theoretical frameworks rely on Rogers' diffusion of innovations theory [3-5]. Rogers defined diffusion as the process by which an innovation disseminates through certain channels over time among members of a social system [3]. In the decision to prescribe a new drug, doctors have to strike a balance between possible benefits and risks. Because new drugs are generally more expensive than established drugs, doctors also have to make this judgement in the wider context of a health service with a limited budget [6]. Within this context, health care providers and formulary decision makers, too, often evaluate newly introduced drugs. Knowledge of the diffusion process would help doctors as well as policy makers in the interpretation of aspects such as effectiveness and economic outcomes of new drugs. The objective of this study was to examine the diffusion of LTG after it became reimbursed in the Netherlands, using a large prescription database over the period from 1996 to 2000. Prescribing trends, usage patterns, and baseline characteristics of patients in a cohort of LTG patients were compared with those of a cohort of patients using the conventional AEDs CBZ, PHT and VPA.

\section{Methods}

\section{Setting}

The prescription data for this study were obtained from the GIP database. This is a project run by the CVZ, an independent advisory and supervisory body in the field of social health insurance. The GIP database contains data from all extramurally prescribed drugs that are dispensed by pharmacists and general practitioners with an in-house pharmacy and are reimbursed by the health insurance funds under the Health Insurance Act. The data were provided by ten health insurance funds and concern 5.6 million compulsorily insured in 2000, which is about $55 \%$ of all compulsorily insured Dutch persons. This sample is representative of the distribution by age and gender of all persons compulsorily insured in the Netherlands.

For each prescription in the GIP database, retrievable information covers the following domains: patient (gender, age and unique anonymous identification number); prescription (trade name, ATC code, dispensing date, dispensed amount and prescribed dose); and prescriber (general practitioner or specialist). The GIP database provides neither the information concerning indications for use of the medicines nor the complete registration of non-prescription medicines.

\section{Study population}

We collected prescription data from the GIP database of all patients who received at least one prescription during the period 1 January 1996 to 31 December 2000 for LTG and/or one of the three most frequently prescribed AEDs in the Netherlands (i.e. CBZ, PHT or VPA; $n=98,043)$. Other AEDs in the Netherlands (i.e. clonazepam, ethosuximide, felbamate, oxcarbazepine, phenobarbital and vigabatrin) represent a combined market share of less than $15 \%$ and were not considered in this study. The date of first prescription of one of the four AEDs was defined as the index date. First-time use was defined as a prescription for one of these four AEDs written during the study period, with no prescription for the same drug having been given during the 12 months before the index date. Only first-time users were included in this study $(n=37,695)$. Due to the selection criteria, there were only first-time users from January 1997 onwards.

In the Netherlands, drugs are dispensed for a maximum of 3 months. In order to prevent the occurrence of information gaps, patients were included only if the period between two subsequent prescriptions (any drug) was less than 180 days $(n=32,206)$.

Thus, the final study population included only new users of one of the four AEDs: either patients who did not receive any AED during the 12 months before the index date or patients who received one or more other AEDs before the index date (Fig. 1).

\section{Analysis of the diffusion process}

The diffusion process of LTG was characterised by analysing different aspects of the prescription pattern. First, the market share of LTG was compared with that of the three conventional AEDs. Market share of the four AEDs was calculated as the number of first-time users of the AED during the study period divided by the source population in the GIP database.

As a second aspect of the diffusion process, differences in the prevalence of baseline characteristics in the LTG cohort and the cohort of patients receiving the conventional AEDs (reference group) were compared. Crude prevalence odds ratios (ORs) were calculated using 


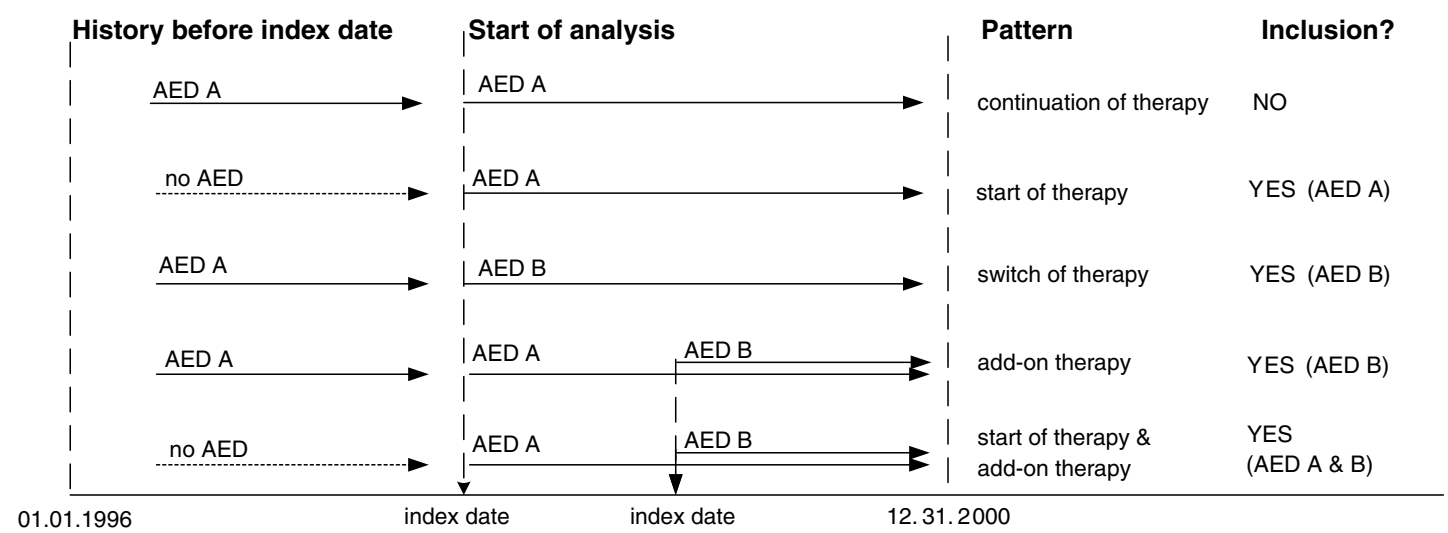

Fig. 1 Study population. $A E D$ antiepileptic drug

multiple logistic regression and were presented with a $95 \%$ confidence interval $(\mathrm{CI})$. The whole group of users of conventional AEDs was taken as reference, instead of users of separate conventional AEDs, because the combined group was considered to be more representative of the entire population using AEDs. In the comparisons between new users of LTG and the reference group, the following characteristics were evaluated:

- Age (on the index date) and gender.

- Type of prescriber: general practitioner, neurologist, psychiatrist or other.

- Prescription of different AEDs during the 12 months before the index date. The prior use of AEDs, and especially that of vigabatrin, was used as a marker for refractory epilepsy.

- Prescription of psychotropic agents (antidepressants, antipsychotics, lithium salts) and migraine-abortive medication during the 12 months before the index date. The use of these drugs was used as a marker for off-label use (e.g. for bipolar depression, migraine) of LTG.

As a consequence of the long follow-up period, patients could be a first-time user of more than one AED (Fig. 1). For the statistical methods used, independent observations are required, which is not the case if a patient contributed more than one observation. Therefore, an analysis was also performed that was restricted to only one new use (chosen randomly) for each user with multiple new uses. This did not change the estimates.

A third aspect of the diffusion process was the analysis of any changes in the population that used LTG throughout the study period. In order to find out whether LTG treatment reached a more heterogeneous population than that considered for inclusion in the initial add-on RCTs [7, 8], trends in the following baseline characteristics were evaluated:

- Increased prevalence of LTG in patients outside the age category $18-65$ years

- Increased prevalence of LTG in patients without a history of AED use

- Increased prevalence of LTG in patients with either lithium or migraine-abortive medication in their history The trend analyses also evaluated whether the prescription patterns regarding gender changed through time. For these analyses, the relative risks (RRs) per period of 3 months were calculated using the first 3 months after the start of reimbursement of LTG as reference. The $\chi^{2}$ test was applied to statistically describe trends in prescribing from 1997 to 2000.

\section{Results}

A total of 29,718 patients who received a prescription of CBZ, PHT, VPA or LTG for the first time during the
Fig. 2 Incidence of antiepileptic drugs in the Netherlands. $C B Z$ carbamazepine, $V P A$ valproate, PHT phenytoin, $L T G$ lamotrigine




study period were identified. These patients accounted for a total of 32,206 new uses of AEDs. The development in market share is presented in Fig. 2. CBZ and VPA accounted for the majority of all new prescriptions (52\% and $29 \%$, respectively). After reimbursement settlement (August 1997), the market share of LTG rose quickly to approximately 9 patients per 100,000 persons insured per year. Initially, the market share of LTG exceeded that of PHT (approximately 7 patients per 100,000), but from 1998 onwards the market share of LTG levelled off to approximately 4.4 patients per 100,000 per year. On average, the market share of LTG was less than $10 \%$; within the group of neurologists, the market share reached $16 \%$.

Table 1 shows the characteristics of the patients, stratified according to the AED treatment started with at the index date. Overall, $60 \%$ of the patients were women, the median age was 52 years. A majority of patients $(76 \%)$ had no history of AEDs in the year before the index date. A high prevalence of psychotropic drugs was registered, especially that of benzodiazepines $(47 \%)$ and antidepressants (24\%). Differences in baseline characteristics of the LTG group and the reference group are presented in Table 2. LTG users were significantly younger than users of one of the conventional AEDs $\left(\mathrm{OR}_{0-17 \text { years }} 3.7 ; 95 \%\right.$ CI 3.3-4.2) and were more often male than female (OR 1.4; 95\% CI 1.3-1.5). LTG patients had more frequently used one or more AED prior to the index date when compared with users of one of the con- ventional AEDs (OR 35.5; 95\% CI 31.6-39.9). Prior use of vigabatrin was significantly more prevalent in the LTG group (OR 25.5; 95\% CI 21.9-29.7). Prescribers of LTG were more often neurologists (OR 2.8; 95\% CI 2.6-3.0). In addition, the prevalence of psychotropic medication and migraine-aborting drugs was significantly lower with users of LTG than with users of conventional AEDs.

Several significant changes were observed in the type of patients receiving LTG during the first years after its introduction (Fig. 3). The ratio of age categories changed; the RR for patients outside of the age category 18-65 years (included in the initial RCTs) increased to 3.0 at the end of the study period ( $P$ value for trend <0.01). The number of patients in the age group 18-64 years fell from $86 \%$ in 1997 to $68 \%$ in 2000. The RR for patients without a history of use of another AED increased to $5.2(P$ value for trend $<0.01)$. The number of patients using LTG without prior use of any AED increased from 3\% in 1997 to $16 \%$ in 2000. Overall, the mean number of AEDs prior to the index date of LTG dropped from 2.2 in 1997 to 1.5 in 2000 . The RR for markers of off-label use increased to 5.0 ( $P$ value for trend <0.01). Over the study period, a significant increase in the number of prescriptions for women was noticed: the RR increased to 1.5 ( $P$ value for trend $<0.01$ ). Differences in the prescription patterns of the conventional AEDs were analysed in a similar way; however, no significant changes were observed for any of the baseline char-
Table 1 Baseline characteristics of the study population

$A E D$ antiepileptic drug, $C B Z$ carbamazepine, $V P A$ valproate, $P H T$ phenytoin, $L T G$ lamotrigine

\begin{tabular}{|c|c|c|c|c|}
\hline \multirow[t]{2}{*}{ Characteristics, $n(\%)$} & \multirow[t]{2}{*}{ LTG $(n=2,944)$} & \multicolumn{3}{|c|}{ Comparator group $(n=29,262)$} \\
\hline & & $\operatorname{CBZ}(n=16,845)$ & VPA $(n=9,267)$ & PHT $(n=3,150)$ \\
\hline \multicolumn{5}{|l|}{ Gender } \\
\hline Male & $1,377(46.8)$ & $6,249(37.1)$ & $3,748(40.4)$ & $1,433(45.5)$ \\
\hline Female & $1,567(53.2)$ & $10,596(62.9)$ & $5,519(59.6)$ & $1,717(54.5)$ \\
\hline \multicolumn{5}{|l|}{ Age } \\
\hline $0-17$ years & $483(16.4)$ & 497 (2.9) & $907(9.8)$ & $58(1.8)$ \\
\hline $18-64$ years & $2,164(73.5)$ & $10,304(61.2)$ & $6,123(66.1)$ & $1,665(52.9)$ \\
\hline$\geq 65$ years & $297(10.1)$ & $6,044(35.9)$ & $2,237(24.1)$ & $1,427(45.3)$ \\
\hline \multicolumn{5}{|l|}{ Prior use of AEDs } \\
\hline None & $341(11.6)$ & $14,779(87.7)$ & 7,094 (76.6) & $2,210(70.2)$ \\
\hline One & $855(29.0)$ & $1,679(10.0)$ & $1,631(17.6)$ & $637(20.2)$ \\
\hline Two & $1,069(36.3)$ & $304(1.8)$ & $400(4.3)$ & $206(6.5)$ \\
\hline Three or more & $679(23.1)$ & $83(0.5)$ & $142(1.5)$ & $97(3.1)$ \\
\hline Vigabatrin & $555(18.9)$ & $77(0.5)$ & $120(1.3)$ & $67(2.1)$ \\
\hline \multicolumn{5}{|l|}{ Prescriber } \\
\hline General practitioner & $506(17.2)$ & $9,489(56.3)$ & $2,241(24.2)$ & $1,051(33.4)$ \\
\hline Neurologist & $1,456(49.4)$ & $2,899(17.2)$ & $3,422(36.9)$ & $1,283(40.7)$ \\
\hline Psychiatrist & $70(2.4)$ & $704(4.2)$ & $818(8.8)$ & $15(0.5)$ \\
\hline Other & $912(31.0)$ & $3,753(22.3)$ & $2,786(30.1)$ & $801(25.4)$ \\
\hline \multicolumn{5}{|l|}{$\begin{array}{l}\text { Prior use of } \\
\text { co-medication }\end{array}$} \\
\hline Antidepressants & $312(10.6)$ & $4,599(27.3)$ & $2,273(24.5)$ & $535(17.0)$ \\
\hline Antipsychotics & $188(6.4)$ & $1,427(8.5)$ & $1,538(16.6)$ & $188(6.0)$ \\
\hline Benzodiazepines & $1,146(38.9)$ & $8,227(48.8)$ & $4,235(45.7)$ & $1,443(45.8)$ \\
\hline Lithium & $65(2.2)$ & $591(3.5)$ & $735(7.9)$ & $11(0.3)$ \\
\hline $\begin{array}{l}\text { Migraine-abortive } \\
\text { drugs }\end{array}$ & $85(2.9)$ & $734(4.4)$ & $1,256(13.6)$ & $89(2.8)$ \\
\hline
\end{tabular}


Table 2 Characteristics of patients starting with lamotrigine compared with those starting with conventional antiepileptic drugs (AEDs)

\begin{tabular}{ll}
\hline Characteristics & OR $(95 \% \mathrm{CI})$ \\
\hline Gender; male & $1.40(1.27-1.48)$ \\
Age & $2.76(2.47-3.09)$ \\
$0-17$ years & Reference \\
$18-64$ years & $0.26(0.23-0.29)$ \\
$\geq 65$ years & \\
Prior use of AEDs & Reference \\
None & $15.30(13.43-17.42)$ \\
One & $82.96(72.22-95.31)$ \\
Two & $148.92(125.60-176.58)$ \\
Three or more & $25.5(21.91-29.71)$ \\
Vigabatrin & \\
Prescriber & $0.21(0.19-0.23)$ \\
General practitioner & Reference \\
Neurologist & $0.24(0.19-0.30)$ \\
Psychiatrist & $0.65(0.59-0.71)$ \\
Others & \\
Prior use of co-medication & \\
Antidepressants & $0.35(0.31-0.39)$ \\
Antipsychotics & $0.57(0.49-0.66)$ \\
Benzodiazepines & $0.70(0.65-0.76)$ \\
Lithium & $0.47(0.37-0.61)$ \\
Migraine-abortive drugs & $0.39(0.31-0.49)$ \\
\hline
\end{tabular}

${ }^{\text {a }}$ Presence versus absence

Reference group is the combined group of patients starting with carbamazepine, phenytoin or valproate. $O R$ odds ratio

acteristics. The same analyses were applied for the reference group; the RRs for the characteristics mentioned above did not change significantly.

\section{Discussion}

Having access to a large prescription database allowed us to follow the diffusion of LTG in a population-based cohort. The results of our study have shown that the uptake of LTG was rather slow. After its introduction in the Netherlands, LTG was prescribed to patients who had previously received other AEDs, suggesting that it was introduced as a second-line or third-line treatment. Subsequently, the diffusion process of LTG resulted in a more heterogeneous population being reached. Accord- ing to Rogers [3] and Ruof et al. [4], new ideas are adopted very slowly during the early stages of the diffusion process; the rate of adoption, however, increases steadily. Remarkably, after the diffusion process for LTG had passed the early stages, a further increase in market share had yet to take place. Several possible barriers can be characterised that probably slowed down the uptake of LTG.

Characteristics of the drug itself affect use Rogers' [3] theory states that the rate of diffusion is inversely proportional to the perceived complexity of the innovation. LTG is not simple to use [9]. The use is hampered by a relatively high incidence of idiosyncratic adverse events, predominantly rash $[10,11]$. Review of trial data showed that severe rashes occur more often with rapid titration in paediatric patients. VPA inhibits the metabolism of LTG, and this has major clinical impact on the risk of skin reactions [9]. The metabolism of LTG is accelerated by enzyme-inducing AEDs such as CBZ and PHT, necessitating higher dosage with these co-medications. The complexity of LTG, i.e. the slow titration schedule and its interaction potential, will probably have caused slower diffusion, expressed in the numbers of new patients. This may possibly have been worsened by the introduction since 2000 of other new AEDs, such as gabapentin and levetiracetam, with less interaction potential and faster titration schedules than LTG. It would be interesting to see whether the diffusion process of these new AEDs was different than that of LTG. Both gabapentin and levetiracetam, however, were not registered in the Netherlands until after 2000, so follow-up data of these drugs are absent from our database.

Prescriber characteristics Individuals do not all adopt an innovation at the same time, and they can be separated into several adopter categories (innovators, early adopters, early majority, late majority and laggards) [3]. The majority of physicians is considered to be conservative (late majority and laggards) regarding drug choice [6]. In our study, we conclude from the differences in baseline characteristics that selective prescribing of LTG to patients with more severe epilepsy has occurred. We believe that prior use of other AEDs, and the use of
Fig. 3 Change in composite index score throughout the study period. $A E D$ antiepileptic drug




vigabatrin especially, can be seen as markers for refractory epilepsy. Selective prescribing, or "channelling", is likely to occur for new representatives of a therapeutic class for which alternatives existed, as is the case for AEDs. This is a general phenomenon, which has been demonstrated for various drug classes, e.g. NSAIDs, antidepressants or spasmolytics [12-14]. The peak in incidence noticed shortly after reimbursement approval could possibly be related to the backlog of patients with refractory epilepsy. About the introduction of LTG, safety issues regarding two other new AEDs played a part. One year after approval of felbamate, reports of aplastic anaemia began to emerge [11]. Several years after the introduction of vigabatrin, evidence was produced that prolonged high-dose treatment may cause severe and symptomatic irreversible visual field constriction [9, 15]. The detection of such safety risks may have withheld many physicians from prescribing AEDs that had been registered after felbamate and vigabatrin.

Economic aspects The pharmaceutical cost of LTG is at least a fivefold that of conventional AEDs. Indiscriminate switching from conventional to new AEDs would have considerable economic implications [9]. A survey among US neurologists showed that neurologists recognise the need to rationalise health care and that they are willing to accept the notion that individual sacrifices can and should be made because of the finite healthcare resources [16]. Increasing pressure on drug budgets will make physicians more reluctant to prescribe new drugs. What's more, the Dutch National Health Care Insurance tried, by issuing the prescription guideline for LTG, to contain the cost of the drug by restricting its prescription to patients with refractory epilepsy. This may also have reduced the "trialability" of LTG. "Potential adopters" want the opportunity to "test" a drug before adopting [3]. The prescription guideline could have prevented this testing, although we were not able to evaluate how the guideline was adhered to in the present study.

Despite the barriers mentioned above, the diffusion of LTG is still ongoing. This study shows that the cohort of LTG users is subject to change. Gradually, the baseline characteristics of the patients start to drift away from those of the patients in the initial, add-on regulatory trials. From 1997 to 2000, LTG gradually became an AED of first and second choice, and was increasingly being given to children and elderly persons. It is wellknown that the publication of new, high-level evidence (i.e. RCTs) influences prescription patterns [17]. Randomised trials comparing LTG to CBZ, PHT and VPA in patients with newly diagnosed epilepsy are available in medical literature [18-21]. Broadly speaking, these trials showed that LTG cannot claim greater efficacy than the conventional AEDs but that the drug seemed to be tolerated better than its comparators, with fewer withdrawals due to adverse events. A similar result emerged from a trial comparing LTG and CBZ in elderly patients (over 65 years of age) with newly diag- nosed epilepsy [22]. Trials and observational studies assessing LTG in the treatment of childhood epilepsy syndromes also became available. This is likely to be an important factor explaining the overall trend towards more use of LTG for the young, as CBZ and PHT are not indicated in the treatment of the idiopathic generalised epilepsy syndromes. Trial data on the effectiveness of LTG in the treatment of diseases other than epilepsy have also started to emerge [23-26]. We noticed a strong increase in markers for off-label use. Our data suggest that there is an increased use of LTG for second-line treatment of bipolar disorder (markers: lithium and antidepressants); neuropathic pain (marker: antidepressants) and migraine (marker: migraine-abortive drugs).

Compared with the conventional AEDs, LTG has been positioned as a better alternative for women of childbearing age, which may explain the increased prevalence in women. There are several aspects that make LTG favourable for this group of patients. First, pregnancy data on LTG increase and the drug does not seem to have major teratogenic effects [27]. Given the known effects of the conventional AEDs, this may possibly explain the increase seen. Second, there is a controversy regarding the question whether the use of VPA is associated with a higher incidence of polycystic ovary syndrome (PCOS) [28, 29]. Herzog and Schacter conclude that, despite limitations in studies reporting an association between the use of VPA and occurrence of PCOS, the evidence cannot be entirely dismissed [30]. As VPA also has teratogenic effects, it may be concluded that it is less suitable for women of childbearing age. LTG has been positioned as a better alternative for this group. Third, LTG lacks an enzyme-inducing capacity and does not reduce the effect of oral contraceptives (whereas CBZ and PHT do). More recently, however, a relevant interaction between LTG and oral contraceptives became known [31].

The changing baseline characteristics shown in Fig. 3 make it clear that there is a gap between the information available from the initial RCTs and the use of LTG in the real world of medicine. These changes are relevant to issues such as rational drug therapy, effectiveness and safety in a population-based setting. This supports postmarketing surveillance studies addressing these issues [32]. The results of this study should be interpreted in the light of its limitations. A first limitation of our study is the lack of additional medical information, most importantly the indications of use. It is common knowledge that AEDs are used for indications other than epilepsy. However, to what extent depends on the individual AED [33]. Using a Dutch prescription database (PHARMO), Shackleton et al. [33] demonstrated that epilepsy was present in $58 \%$ of patients using a single AED, and epilepsy prevalence was $93 \%$ in patients using more than one AED. CBZ was more often used for other indications than the other conventional AEDs. We believe that, by using a combined reference group, we are comparing LTG to a population-based use of conventional AEDs (i.e. use of these drugs for 
epilepsy and other indications as prescribed by various types of physicians). We had to use surrogate markers, however, to illustrate off-label use of LTG. The prevalence of a marker in an individual patient will not always mean that off-label use is the case, for some misclassification has certainly occurred.

Furthermore, the GIP database comprises prescription data of the compulsorily insured patients in the Netherlands. It might be argued whether the results are also representative of the higher socio-economic classes, which are not covered by the national health insurance system. However, as there are no reimbursement limitations for antidepressants in either the national health insurance system or the private insurance companies, differences between socio-economic classes are not expected. Another limitation is that we were not able to evaluate other relevant characteristics that enhanced diffusion, such as the physician's attitude towards new drugs, such as LTG, or the impact of drug marketing by the pharmaceutical company. The latter aspect may also be an explanation for the increased use of LTG for women; however, we were not able to evaluate this.

Our conclusion is that there are a multitude of factors influencing the diffusion of LTG into daily practice. Starting in a selected group of patients with severe epilepsy, the drug gradually diffuses to a much more heterogeneous population. Understanding the process of diffusion is important in the evaluation of the place in therapy of LTG, its effectiveness in real life and its cost consequences.

Acknowledgements This study was part of a research project on the rational use of lamotrigine. This project has been subsidised by the Dutch National Health Care Insurance Board. Neither the Health Care Insurance Board nor any other organisation exerted undue influence with regard to the results of this study. The authors have no financial ties with any pharmaceutical company.

\section{References}

1. Chadwick D (1998) Do new antiepileptic drugs justify their expense? Arch Neurol 55(8):1140-1142

2. Dichter MA, Brodie MJ (1996) New antiepileptic drugs. N Engl J Med 334(24):1583-1590

3. Rogers EM (2003) Diffusion of innovations. Free Press, New York

4. Ruof J, Mittendorf T, Pirk O, Graf von der Schulenburg JM (2002) Diffusion of innovations: treatment of Alzheimer's disease in Germany. Health Policy 60:59-66

5. Sambamoorthi U, Olfson M, Walkup JT, Crystal S (2003) Diffusion of new generation antidepressant treatment among elderly diagnosed with depression. Med Care 41(1):180-194

6. Jones MI, Greenfield SM, Bradley CP (2001) Prescribing new drugs: qualitative study of influences on consultants and general practitioners. BMJ 323:378

7. Matsuo F, Bergen D, Faught E, Messenheimer JA, Dren AT, Rudd GD et al (1993) Placebo-controlled study of the efficacy and safety of lamotrigine in patients with partial seizures. Neurology 43:2284-2291
8. Messenheimer J, Ramsay RE, Willmore LJ, Leroy RF, Zielinski JJ, Mattson R et al (1994) Lamotrigine therapy for partial seizures: a multicenter, placebo- controlled, doubleblind, cross-over trial. Epilepsia 35(1):113-121

9. Chadwick D (1999) The use of new antiepileptic drugs. J R Coll Physicians Lond 33:328-332

10. Deckers CLP, Knoester PD, de Haan GJ, Keyser A, Renier WO, Hekster YA (2003) Selection criteria for the clinical use of the newer antiepileptic drugs. CNS Drugs 17(6):405-421

11. LaRoche SM, Helmers SL (2004) The new antiepileptic drugs. JAMA 291(5):605-614

12. Leufkens HGM, Urquhart J, Stricker BHCh, Bakker A, Petri H (1992) Channelling of controlled release formulation of ketoprofen (Oscorel) in patients with history of gastro-intestinal problems. J Epidemiol Community Health 46:428-432

13. Egberts AC, Lenderink AW, De Koning FH, Leufkens HG (1997) Channeling of three newly introduced antidepressants to patients not responding satisfactorily to previous treatment. J Clin Psychopharmacol 17(3):149-155

14. Movig KLL, Egberts ACG, Lenderink AW, Leufkens HGM (2000) Selective prescribing of spasmolytics. Ann Pharmacother 34(6):716-720

15. Eke T, Talbot JF, Lawden MC (1997) Severe persistent visual field constriction associated with vigabatrin. BMJ 314(7075):180-181

16. Holloway RG, Ringel SP, Bernat JL, Keran CM, Lawyer BL (2000) US neurologists: attitudes on rationing. Neurology 55(10):1492-1497

17. Calvo CB, Rubinstein A (2002) Influence of new evidence on prescription patterns. J Am Board Fam Pract 15:457-462

18. Brodie MJ, Richens A, Yuen AW (1995) Double-blind comparison of lamotrigine and carbamazepine in newly diagnosed epilepsy. UK Lamotrigine/Carbamazepine Monotherapy Trial Group. Lancet 345(8948):476-479

19. Reunanen M, Dam M, Yuen AW (1996) A randomised open multicentre comparative trial of lamotrigine and carbamazepine as monotherapy in patients with newly diagnosed or recurrent epilepsy. Epilepsy Res 23(2):149-155

20. Steiner TJ, Dellaportas CI, Findley LJ, Gross M, Gibberd FB, Perkin GD et al (1999) Lamotrigine monotherapy in newly diagnosed untreated epilepsy: a double- blind comparison with phenytoin. Epilepsia 40(5):601-607

21. Nieto-Barrera M, Brozmanova M, Capovilla G, Christe W, Pedersen B, Kane K et al (2001) A comparison of monotherapy with lamotrigine or carbamazepine in patients with newly diagnosed partial epilepsy. Epilepsy Res 46(2):145-155

22. Brodie MJ, Overstall PW, Giorgi L (1999) Multicentre, doubleblind, randomised comparison between lamotrigine and carbamazepine in elderly patients with newly diagnosed epilepsy. The UK Lamotrigine Elderly Study Group. Epilepsy Res 37(1):81-87

23. Steiner TJ, Findley LJ, Yuen AW (1997) Lamotrigine versus placebo in the prophylaxis of migraine with and without aura Cephalalgia 17(2):109-112

24. Eisenberg E, Lurie Y, Braker C, Daoud D, Ishay A (2001) Lamotrigine reduces painful diabetic neuropathy: a randomized, controlled study. Neurology 57(3):505-509

25. Barbosa L, Berk M, Vorster M (2003) A double-blind, randomized, placebo-controlled trial of augmentation with lamotrigine or placebo in patients concomitantly treated with fluoxetine for resistant major depressive episodes. J Clin Psychiatry 64(4):403-407

26. Bowden CL, Calabrese JR, Sachs GS, Asghar SA, Hompland M, Montgomery P et al. (2003) A placebo-controlled 18-month trial of lamotrigine and lithium maintenance treatment in recently manic or hypomanic patients with bipolar I disorder. Arch Gen Psychiatry 60(4):392-400

27. Tennis P, Eldridge RR (2002) Preliminary results on pregnancy outcomes in women using lamotrigine. Epilepsia 43(10):11611167 
28. Isojarvi JI, Rattya J, Myllyla VV, Knip M, Koivunen R, Pakarinen AJ et al (1998) Valproate, lamotrigine, and insulinmediated risks in women with epilepsy. Ann Neurol 43(4):446451

29. Genton P, Bauer J, Duncan S, Taylor AE, Balen AH, Eberle A et al. (2001) On the association between valproate and polycystic ovary syndrome. Epilepsia 42(3):295-304

30. Herzog AG, Schachter SC (2001) Valproate and the polycystic ovarian syndrome: final thoughts. Epilepsia 42(3):311-315
31. Sabers A, Ohman I, Christensen J, Tomson T (2003) Oral contraceptives reduce lamotrigine plasma levels. Neurology 61(4):570-571

32. French JA (2002) Postmarketing surveillance of new antiepileptic drugs: the tribulations of trials. Epilepsia 43(9):951-955

33. Shackleton DP, Westendorp RG, Kasteleijn-Nolst Trenite DG, de Boer A, Herings RM (1997) Dispensing epilepsy medication: a method of determining the frequency of symptomatic individuals with seizures. J Clin Epidemiol 50(9):1061-1068 